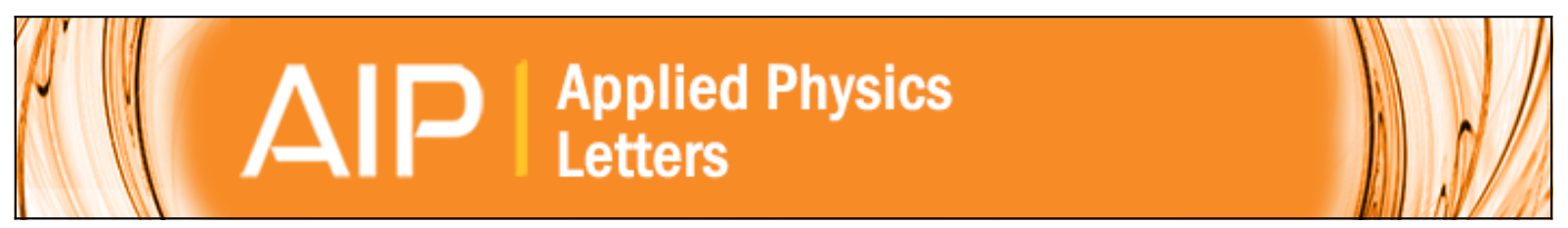

\title{
A smart microelectromechanical sensor and switch triggered by gas
}

Adam Bouchaala, Nizar Jaber, Osama Shekhah, Valeriya Chernikova, Mohamed Eddaoudi, and Mohammad I.

Younis

Citation: Applied Physics Letters 109, 013502 (2016); doi: 10.1063/1.4955309

View online: http://dx.doi.org/10.1063/1.4955309

View Table of Contents: http://scitation.aip.org/content/aip/journal/apl/109/1?ver=pdfcov

Published by the AIP Publishing

\section{Articles you may be interested in}

Si membrane based tactile sensor with active matrix circuitry for artificial skin applications

Appl. Phys. Lett. 106, 043502 (2015); 10.1063/1.4906373

Zero displacement microelectromechanical force sensor using feedback control

Appl. Phys. Lett. 104, 153502 (2014); 10.1063/1.4871380

Design and fabrication of a CMOS-compatible MHP gas sensor

AIP Advances 4, 031339 (2014); 10.1063/1.4869616

Dynamics of electrostatic microelectromechanical systems actuators

J. Math. Phys. 53, 022703 (2012); 10.1063/1.3684748

Smart optical microscanner with piezoelectric resonator, sensor, and tuner using $\mathrm{Pb}$ ( $\mathrm{Zr}, \mathrm{Ti}$ ) $\mathrm{O} 3$ thin film Appl. Phys. Lett. 90, 183514 (2007); 10.1063/1.2736296

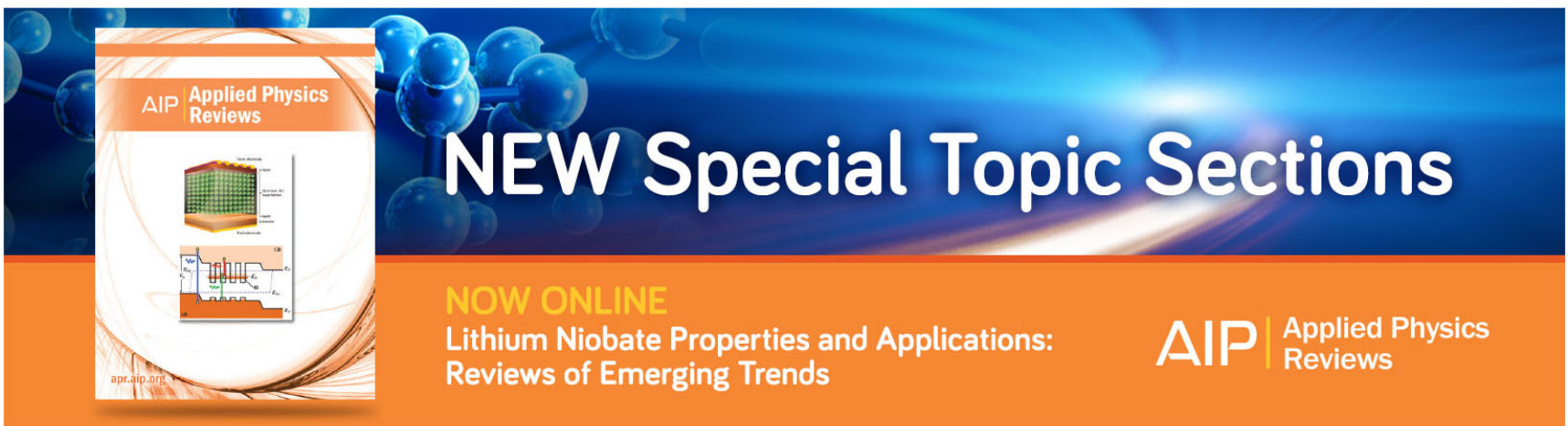




\title{
A smart microelectromechanical sensor and switch triggered by gas
}

\author{
Adam Bouchaala, Nizar Jaber, Osama Shekhah, Valeriya Chernikova, Mohamed Eddaoudi, \\ and Mohammad I. Younis ${ }^{\text {a) }}$ \\ Physical Sciences and Engineering Division, King Abdullah University of Science and Technology, \\ Thuwal 23955-9600, Saudi Arabia
}

(Received 18 March 2016; accepted 23 June 2016; published online 5 July 2016)

\begin{abstract}
There is an increasing interest to realize smarter sensors and actuators that can deliver a multitude of sophisticated functionalities while being compact in size and of low cost. We report here combining both sensing and actuation on the same device based on a single microstructure. Specifically, we demonstrate a smart resonant gas (mass) sensor, which in addition to being capable of quantifying the amount of absorbed gas, can be autonomously triggered as an electrical switch upon exceeding a preset threshold of absorbed gas. Toward this, an electrostatically actuated polymer microbeam is fabricated and is then functionalized with a metal-organic framework, namely, HKUST-1. The microbeam is demonstrated to absorb vapors up to a certain threshold, after which is shown to collapse through the dynamic pull-in instability. Upon pull-in, the microstructure can be made to act as an electrical switch to achieve desirable actions, such as alarming. Published by AIP Publishing. [http://dx.doi.org/10.1063/1.4955309]
\end{abstract}

Micro and nano-resonators have been shown to be a promising platform for the detection and recognition of biological elements and chemical gases. ${ }^{1,2}$ Different types of microstructures, such as cantilever and clamped-clamped microbeams, have been employed for resonance sensing. ${ }^{3-6}$ Classically, the shift of the natural frequency of a microstructure is tracked to determine the amount of adsorbed mass attached on the functionalized surface. As the demand for smarter sensors grows larger, new techniques for mass detection have emerged. Nonlinear-dynamics based gas sensors have shown outstanding potential to detect accurately an added mass. ${ }^{7-10}$ Kumar et al. ${ }^{7}$ recently demonstrated a bifurcation-based mass sensor using the softening behavior of a piezoelectrically actuated cantilever. Dai et al. ${ }^{8}$ emphasized the advantages of using the nonlinear response of a nanomechanical device for mass sensing.

In parallel to the developments of Microsensors, Microand Nano-switches, especially Radio Frequency (RF) switches, have been researched and proposed for various applications. ${ }^{11-14}$ These switches are based on a nonlinear static instability in parallel-plate capacitors, pull-in, in which the movable electrode of the capacitor collapses into the other stationary one by a DC voltage load. ${ }^{15}$ This collapse closes an electric circuit as a switch.

Unlike RF switches, resonant mass sensors rely on a DC load $V_{D C}$ superimposed to an $\mathrm{AC}$ harmonic load $V_{A C}$ to drive the microstructure into resonance. ${ }^{16}$ Recent research has shown that if $V_{D C}$ and $V_{A C}$ are increased beyond certain limits, dynamic pull-in instability can occur leading to the collapse of the microbeam. ${ }^{17-19}$ More interestingly, for certain ranges of $V_{D C}$ and $V_{A C}$, an instability frequency band is born, pull-in band, wherein the microbeam is forced to collapse if operated within this band. In the classical sense, this dynamic instability is viewed as destructive to resonant sensors, and hence, is undesirable and needs to be avoided.

\footnotetext{
${ }^{\text {a) }}$ Author to whom correspondence should be addressed. Electronic mail: Mohammad.younis@kaust.edu.sa
}

Dynamic pull-in and the pull-in band form the basis of the switch principle of this work. The basic principle is illustrated in Fig. 1, where a stable resonator is excited into resonance at a fixed frequency outside the pull-in band. Upon gas detection exceeding a certain threshold, the natural frequency of the beam, and accordingly its pull-in band, shifts to lower value making the operating frequency lying inside it. Hence, the beam then collapses as a switch. This is further clarified in Fig. 1(a), which compares the time history of the beam before pull-in (stable response) to that when pulls-in (diverging unstable response).

The experimental investigation starts with the fabrication of an electrostatically actuated clamped-clamped beam of length $700 \mu \mathrm{m}$ with a two-third electrode configuration. ${ }^{20}$ The partial electrode configuration aims to electrically separate the upper electrode (the beam) from the lower electrodes upon collapsing so that the beam can survive and to allow repeatable experiments. Also, the two-third electrode can be used to excite the third mode of the beam, if needed, to increase the sensitivity of mass detection. ${ }^{20}$ A silicon dioxide layer for insulation is deposited on the 4-in. silicon wafer. Then, a gold/chrome layer of thicknesses $250 \mathrm{~nm} / 50 \mathrm{~nm}$ is sputtered to form the lower electrode. The sacrificial layer is formed of amorphous silicon, which is deposited on top of the gold and chrome layers. The lower electrode is connected to the upper electrode through two anchors etched in the sacrificial layer. The upper electrode is formed by chrome/gold/ chrome layers of thicknesses $50 \mathrm{~nm} / 250 \mathrm{~nm} / 50 \mathrm{~nm}$. In order to form the structural layer, $6 \mu \mathrm{m}$ thickness layer of polyimide is spun and cured at gradually increasing temperature from $150{ }^{\circ} \mathrm{C}$ to $350{ }^{\circ} \mathrm{C}$ for $50 \mathrm{~min}$ and then held at $350{ }^{\circ} \mathrm{C}$ for $30 \mathrm{~min}$. Finally, to protect the microbeam during the reactive ion etching, a $50 \mathrm{~nm}$ nickel layer is sputtered on the top surface of the structural layer (Fig. 2).

Next, to investigate the resolution of the smart switch, a noise analysis is performed. ${ }^{21}$ We start by measuring the phase variation in time at a fixed frequency. For a fixed operating frequency, phase variation with time can be related to 

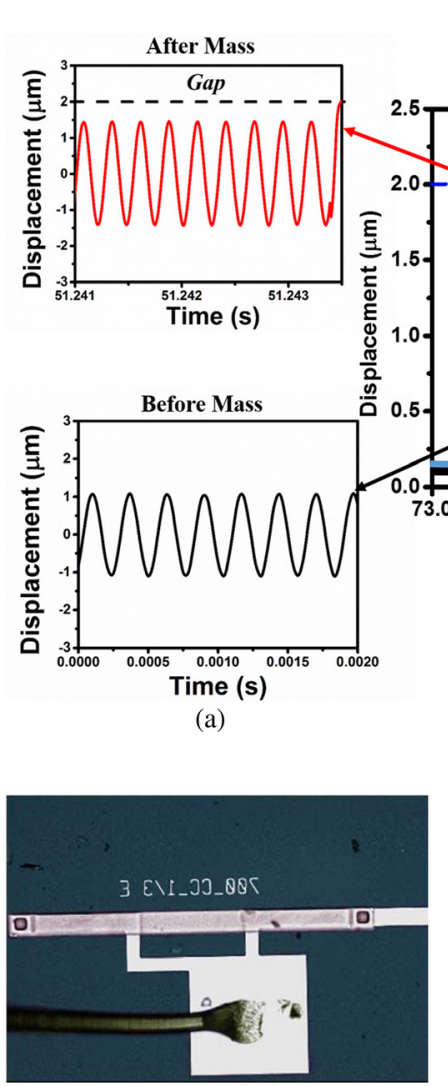

(a)

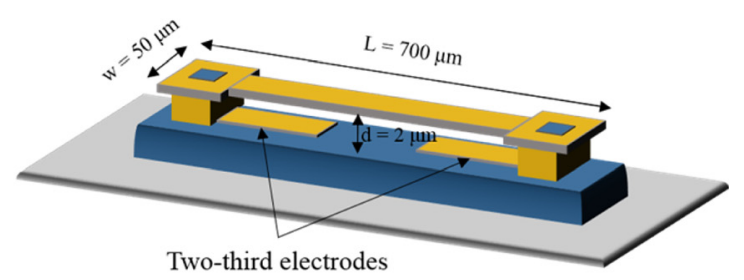

(b) the thermal fluctuations around the resonator, which are considered a key factor for resonant sensors that determines their capabilities and limitations. There are also several other sources that can contribute to noise, such as the flicker noise, which is inversely proportional to the frequency and is especially troublesome at low frequencies, the extrinsic noise due to the experimental setup, and the electrical noise from the electrical connections. Here, due to the limitations of the impedance analyzer that does not allow an AC voltage above $1 \mathrm{~V}$, we conduct the noise analysis on a voltage load of $V_{A C}=1 \mathrm{~V}$ and $V_{D C}=15 \mathrm{~V}$. This differs from the latter used voltage load of $V_{A C}=16 \mathrm{~V}$ and $V_{D C}=1 \mathrm{~V}$. Since both lead to almost the same harmonic electrostatic amplitude of ( $\left.2 V_{A C} V_{D C}\right)$, this should lead to close results.

A precision impedance analyzer Agilent 4294A is connected to the microbeam in order to measure the phase fluctuations. The constant excitation frequency is selected in order to be in the linearly fitted zone (Fig. 3(a)). The phase evolution in time at a constant excitation frequency $f=73.1 \mathrm{kHz}$ is plotted in Fig. 3(b), from which the phase variation is determined to be $d \phi=0.1^{\circ}$. Using the calculated slope $|d \phi / d f|=0.0016\left({ }^{\circ} / \mathrm{Hz}\right)$, one can determine the minimum detectable frequency $\delta f_{\text {noise }}=d \phi /|d \phi / d f| \approx 60(\mathrm{~Hz})$. To determine the minimum detectable mass of the microbeam, the sensor resolution is calculated using

$$
\Re^{-1}=\left|\frac{d m}{d f}\right|=\frac{2 m_{e f f}}{f_{\text {res }, 0 V}},
$$

where $m_{\text {eff }}=236 \mathrm{ng}$ is the effective mass ${ }^{22}$ of the microbeam and $f_{\text {res, } O V}=79 \mathrm{kHz}$ is the natural frequency at $V_{D C}=0 \mathrm{~V}$. From Eq. (1), the responsivity of the microbeam is $\Re^{-1} \approx 6(\mathrm{pg} / \mathrm{Hz})$. It follows that the minimum detectable mass can be expressed as $\delta m_{\text {noise }}=\Re^{-1} \delta f_{\text {noise }}$, which is found equal $\delta m_{\text {noise }}=360 \mathrm{pg}$.

The microbeam is functionalized with HKUST-1, which is a Metal-Organic Framework (MOF) that is considered a very promising chemical layer for gas absorption compared

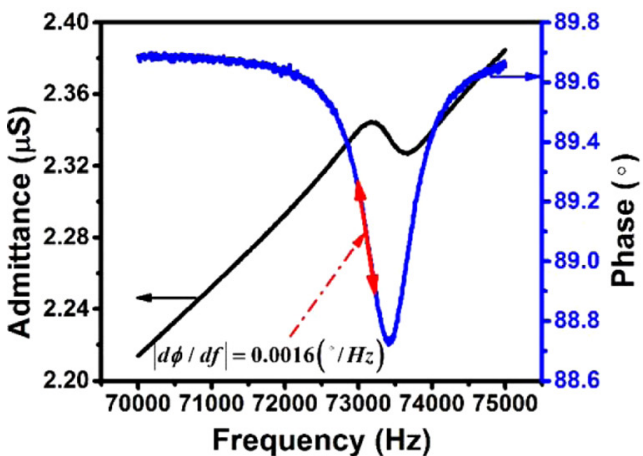

(a)

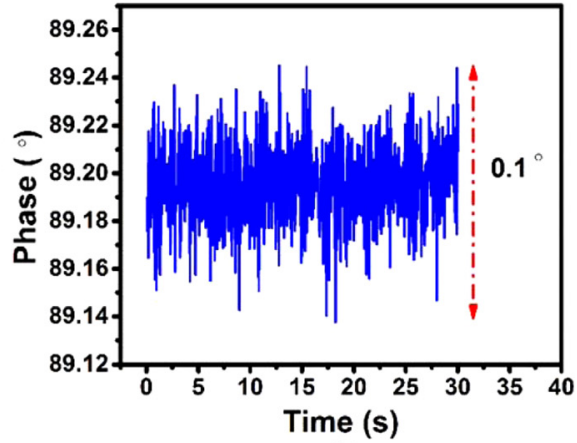

(b)
FIG. 3. Noise analysis at a voltage load of $V_{D C}=15 \mathrm{~V}$ and $V_{A C}=1 \mathrm{~V}$ and at a pressure of 3.3 Torr. (a) Admittance and phase as a function of frequency near resonance. (b) Variation of the phase in time at $f=73.1 \mathrm{kHz}$. 


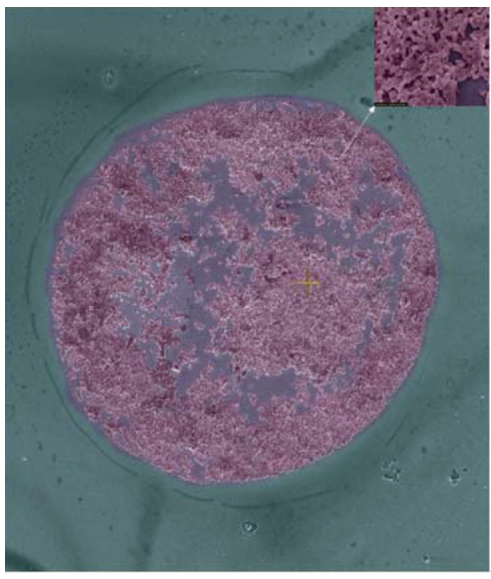

(a)

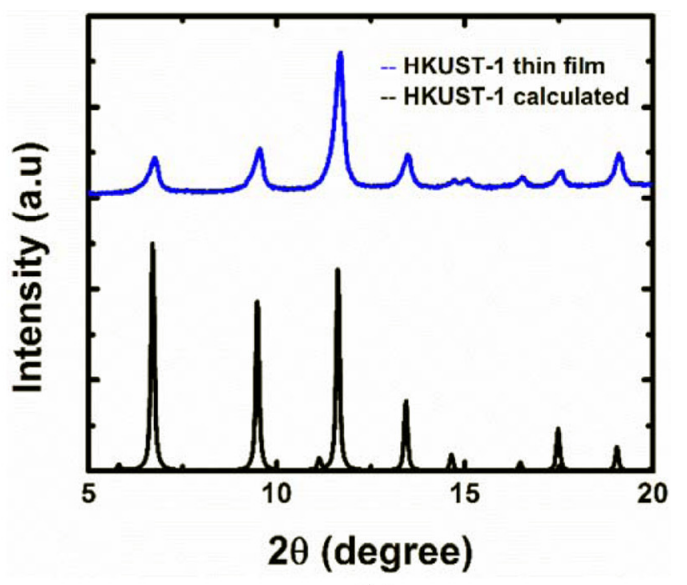

(b)
FIG. 4. (a) An SEM top view image of an HKUST-1 Metal-Organic Framework droplet after deposition on the beam surface. (b) A PXRD pattern of the MOF droplet on the beam (blue) and calculated (black). to traditional organics and polymers. ${ }^{23-27}$ Drop-on-demand inkjet printer with a nozzle of $20 \mu \mathrm{m}$ diameter is used to coat the microstructure. Fig. 4(a) shows a scanning electron micrograph (SEM) of the HKUST-1 MOF droplet after deposited on the beam surface. In order to ascertain the existence of the MOF on top of the microbeam surface, powder X-ray diffraction (PXRD) is performed. Fig. 4(b) confirms the formation of the HKUST-1 MOF structure as concluded from the excellent agreement of the HKUST-1 thin film PXRD (Figure 4(b) blue line), with the bulk HKUST-1 MOF calculated PXRD pattern (Figure 4(b) black). ${ }^{28}$

The experimental setup for vapor sensing is shown schematically in Fig. 5. A scanning laser Doppler vibrometer MSA-500 from Polytec, Inc., ${ }^{29}$ is used for optical measurement in order to demonstrate the pull-in phenomenon. A customized test chamber is designed and connected to the test hardware. A high-pressure nitrogen supply is connected to a bubbler, which contains the desired gas in the liquid phase. The output of the bubbler is connected to the test chamber via the flow controller. A data acquisition card is connected to the flow controller and is set to allow $0.41 / \mathrm{min}$ of the ethanol vapor flow, which leads to a pressure of 3.3 Torr.

Fig. 6(a) represents the measured frequency response of the microbeam for $V_{\mathrm{DC}}=1 \mathrm{~V}$ and $V_{\mathrm{AC}}=16 \mathrm{~V}$ at 3.3 Torr. Fig. 6(b) shows a close up for the pull-in band, which is $\Delta f=520 \mathrm{~Hz}$. These figures have been obtained after raising the exciting voltage load gradually so that the dynamic response goes from linear behavior to hardening behavior, and then to the dynamic pull-in regime.

To demonstrate the concept of the switch triggered by gas, the operating frequency needs to be selected in a safe stable regime outside the pull-in band. The limits of this regime are determined according to the noise analysis. The upper limit of the operating frequency range depends on

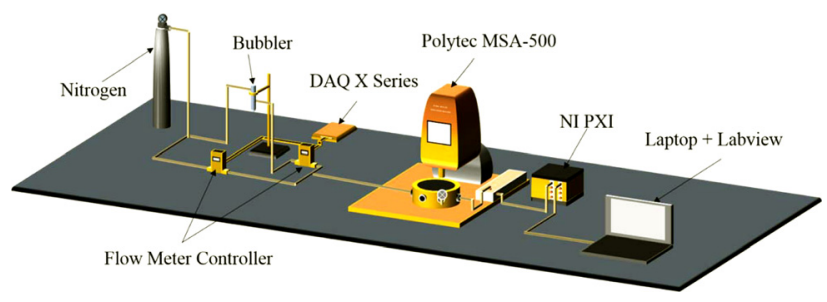

FIG. 5. A schematic of the gas sensing setup. the quantity of mass that can be stored in the MOF. The operating frequency $f_{\text {Operating }}=75.01 \mathrm{kHz}$ is selected for the experiments, as shown in Figs. 6(a) and 6(b) (point A), which is located at $\delta f=87 \mathrm{~Hz}$ away from the start of the pull-in band (point B). Although the noise analysis is performed at a different excitation force and at $73.1 \mathrm{kHz}$, we found out experimentally that increasing the AC voltage from $1 \mathrm{~V}$ to $16 \mathrm{~V}$ decreases the phase fluctuations. Hence, the minimum detectable frequency in the gas sensing experiment at $75.01 \mathrm{kHz}$ is much less than $60 \mathrm{~Hz}$.

Fig. 6(c) shows the gradual increase in the midpoint displacement of the microbeam during the ethanol vapor exposure. This increase in displacement is due to the shift of the pull-in band toward the operating frequency. It can be viewed equivalently as sliding the operating frequency to higher values toward a fixed pull-in band (riding the upper curve of Fig. 6(a)). After $52 \mathrm{~s}$, the displacement suddenly increases exceeding the gap width $(2 \mu \mathrm{m})$ between the two electrodes after a certain mass threshold, thus indicating a pull-in event where the beam hits the lower electrode. Fig. 6(d) confirms the operating frequency of the time history of Fig. 6(c) through calculating its Fast Fourier Transform, FFT.

To quantify the absorbed mass during the amplitude increase, and also at the event of pull-in, we linearly curve-fit the upper branch of the frequency response curve of Fig. 6(a) before pull-in. The slope is found to be $|d Y / d f|=3.95$ $\times 10^{-3}(\mu \mathrm{m} / \mathrm{Hz})$. Based on the slope and the starting operating frequency, one can determine the frequency shift, and hence the absorbed mass, from the measured amplitude. For example, by measuring the difference in amplitude just before the pull-in and the initial displacement value at the operating point, $\Delta Y=0.353 \mu \mathrm{m}$, it is estimated that the frequency shift to reach pull in is $\Delta f=89.4 \mathrm{~Hz}$ corresponding to an added mass threshold of $\Delta m=536 \mathrm{pg}$. Note that the calculated frequency shift is close to the actual value of $87 \mathrm{~Hz}$ (3\% error). The added mass threshold can be controlled by shifting the operating frequency to lower or higher values. For example, to decrease the mass threshold value, the operating frequency can be moved closer to the pull-in band up to the limit the noise permits $\left(\delta f_{\text {noise }}=60 \mathrm{~Hz}\right.$ ).

In conclusion, we proposed a device that has the potential to combine sensing and switching functionalities in a 


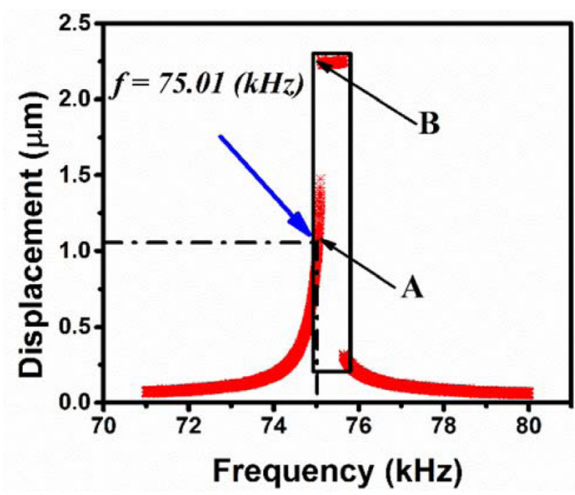

(a)

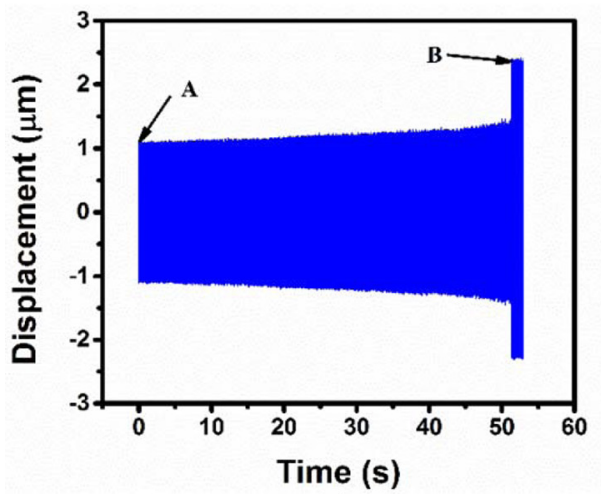

(c)

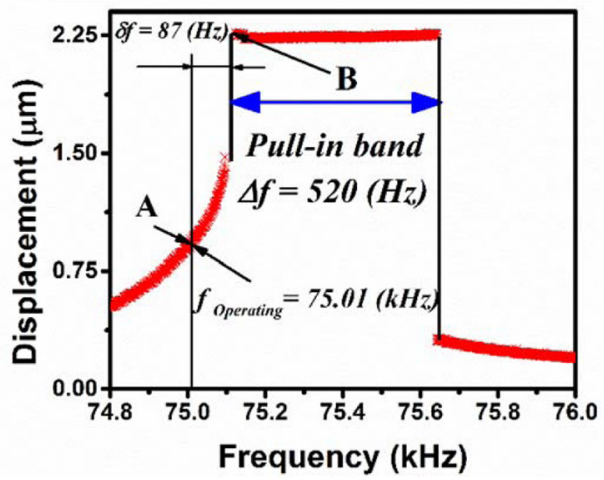

(b)

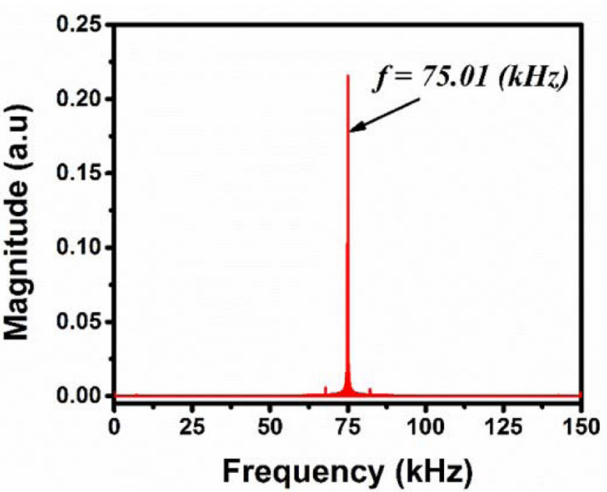

(c)
FIG. 6. Gas sensing experiment. (a) Frequency response near the pull-in band before vapor exposure with $f=75.01 \mathrm{kHz}$ selected as the operating frequency. (b) An enlarged view of the frequency response of Fig. 6(a) to show the pull-in band. (c) Time history of the midpoint displacement of the beam during vapor exposure. (d) FFT of the time history. single device excited near pull-in. This smart platform is configured in order to be triggered after a certain threshold, which is related to the selected operating frequency. An approximate technique to quantify the captured mass as the displacement increases before pull-in has been developed. The accuracy of this technique remains in question as the behavior before pull-in may not necessarily result in the near linear frequency response upper branch as the one shown in this letter. Hence, more studies are needed to be conducted to examine this aspect. In addition, the demonstrated device requires operation at a reduced pressure, which puts limitations on its practical use. Hence, operating the devices at atmospheric pressures needs to be investigated in the future. This study has shown a proof-of-concept experiment of how to control and trigger the pull-in event upon mass detection. With a specially designed circuit, this action can be used to close an electric circuit as a switch and thus deliver a desirable functionality, such as alarming.

${ }^{1}$ T. Thundat, E. Wachter, S. Sharp, and R. Warmack, Appl. Phys. Lett. 66, 1695 (1995).

${ }^{2}$ R. T. Howe and R. S. Muller, IEEE Trans. Electron Devices 33, 499 (1986).

${ }^{3}$ M. Hanay, S. Kelber, A. Naik, D. Chi, S. Hentz, E. Bullard, E. Colinet, L. Duraffourg, and M. Roukes, Nat. Nanotechnol. 7, 602 (2012).

${ }^{4}$ M. Su, S. Li, and V. P. Dravid, Appl. Phys. Lett. 82, 3562 (2003).

${ }^{5}$ A. Gupta, D. Akin, and R. Bashir, Appl. Phys. Lett. 84, 1976 (2004).

${ }^{6}$ L. Senesac and T. G. Thundat, Mater. Today 11, 28 (2008).

${ }^{7}$ V. Kumar, J. W. Boley, Y. Yang, H. Ekowaluyo, J. K. Miller, G. T.-C. Chiu, and J. F. Rhoads, Appl. Phys. Lett. 98, 153510 (2011).

${ }^{8}$ M. D. Dai, K. Eom, and C.-W. Kim, Appl. Phys. Lett. 95, 203104 (2009).

${ }^{9}$ T. Hiller, L. L. Li, E. L. Holthoff, B. Bamieh, and K. L. Turner, J. Microelectromech. Syst. 24, 1275 (2015).
${ }^{10}$ A. Bouchaala, N. Jaber, O. Yassine, O. Shekhah, V. Chernikova, M. Eddaoudi, and M. Younis, Sensors 16, 758 (2016).

${ }^{11}$ S. W. Lee, S. J. Park, E. E. Campbell, and Y. W. Park, Nat. Commun. 2, $220(2011)$

${ }^{12}$ J. O. Lee, Y.-H. Song, M.-W. Kim, M.-H. Kang, J.-S. Oh, H.-H. Yang, and J.-B. Yoon, Nat. Nanotechnol. 8, 36 (2013).

${ }^{13}$ W. W. Jang, J. O. Lee, J.-B. Yoon, M.-S. Kim, J.-M. Lee, S.-M. Kim, K.-H. Cho, D.-W. Kim, D. Park, and W.-S. Lee, Appl. Phys. Lett. 92, 103110 (2008).

${ }^{14}$ X. Feng, M. Matheny, C. Zorman, M. Mehregany, and M. Roukes, Nano Lett. 10, 2891 (2010).

${ }^{15} \mathrm{M}$. I. Younis, MEMS linear and nonlinear statics and dynamics (Springer, New York, 2011).

${ }^{16}$ T. P. Burg, M. Godin, S. M. Knudsen, W. Shen, G. Carlson, J. S. Foster, K. Babcock, and S. R. Manalis, Nature 446, 1066 (2007).

${ }^{17}$ M. I. Younis and F. Alsaleem, J. Comput. Nonlinear Dyn. 4, 021010 (2009).

${ }^{18}$ A. H. Nayfeh, M. I. Younis, and E. M. Abdel-Rahman, Nonlinear Dyn. 48, 153 (2007).

${ }^{19}$ F. M. Alsaleem, M. I. Younis, and L. Ruzziconi, J. Microelectromech. Syst. 19, 794 (2010).

${ }^{20}$ N. Jaber, A. Ramini, A. A. Carreno, and M. I. Younis, J. Micromech. Microeng. 26, 025008 (2016)

${ }^{21}$ J. Teva, G. Abadal, F. Torres, J. Verd, F. Perez-Murano, and N. Barniol, Ultramicroscopy 106, 808 (2006).

${ }^{22}$ B. Hauer, C. Doolin, K. Beach, and J. Davis, Ann. Phys. 339, 181 (2013).

${ }^{23}$ H. Li, M. Eddaoudi, M. O'Keeffe, and O. M. Yaghi, Nature 402, 276 (1999).

${ }^{24}$ P. Nugent, Y. Belmabkhout, S. D. Burd, A. J. Cairns, R. Luebke, K. Forrest, T. Pham, S. Ma, B. Space, L. Wojtas, M. Eddaoudi, and M. J. Zaworotko, Nature 495, 80 (2013).

${ }^{25}$ O. Shekhah, J. Liu, R. A. Fisher, and C. Wöll, Chem. Soc. Rev. 44, 1081 (2011).

${ }^{26}$ O. Shekhah, N. Roques, V. Mugnaini, C. Munuera, C. Ocal, J. Veciana, and C. Wöll, Langmuir 24, 6640 (2008).

${ }^{27}$ E. M. S. Azzam, A. Bashir, O. Shekhah, A. R. E. Alawady, A. Birkner, C. Grunwald, and C. Wöll, Thin Solid Films 518, 387 (2009).

${ }^{28}$ P. St. Petkov, G. N. Vayssilov, J. Liu, O. Shekhah, Y. Wang, C. Wöll, and T. Heine, ChemPhysChem 13, 2025 (2012).

${ }^{29}$ See www.polytec.com/us for POLYTEC. 